\title{
FE SOLUTIONS OF NEAR-TIP FIELD FOR MODE-I CRACKS WITH RESIDUAL SURFACE TENSION
}

\author{
Jaroon Rungamornrat ${ }^{1}$, Son Ngoc Nguyen ${ }^{2}$, Supawat Wongthongsiri ${ }^{3}$, \\ Keerati Panupattanapong ${ }^{4}$, Teerapong Senjuntichai ${ }^{* *}$, and Thai Binh Nguyen ${ }^{6}$ \\ ${ }^{1-5}$ Applied Mechanics and Structures Research Unit, Department of Civil Engineering, Faculty of \\ Engineering, Chulalongkorn University, Bangkok, Thailand, e-mail: Jaroon.R@ chula.ac.th ${ }^{1}$, \\ nguyenson2311@gmail.com ${ }^{2}$, artsupawat@hotmail.com ${ }^{3}$, keeratibs@gmail.com ${ }^{4}$, Teerapong.S@ chula.ac.th ${ }^{5}$ \\ ${ }^{6}$ Department of Mechanics of Materials and Structures, Faculty of Civil Engineering, HCMC University of \\ Technology, Ho Chi Minh City, Vietnam, e-mail: tbnguyen@hcmut.edu.vn ${ }^{6}$
}

Received Date: May 8, 2019; Revised Date: September 9, 2019; Acceptance Date: September 10, 2019

\begin{abstract}
This paper presents finite element solutions of a near-tip elastic field of a straight, nano-scale crack in a two-dimensional, linear elastic, whole plane subjected to mode-I crack-face loads. The mathematical model is formulated using a continuum-based theory of classical linear elasticity together with Gurtin-Murdoch surface model to capture the role of the residual surface tension present on the crack-face material layer. The formulation finally yields a second-order, integrodifferential equation governing the crack opening displacement. A weighted residual technique along with the regularization procedure is applied to establish a weakly singular weak-form equation with the involved kernel of $\mathcal{O}(\ln r)$. Galerkin strategy and the finite element procedure are then employed to discretize the weak-form equation. Various types of element shape functions, generated by standard $C^{0}$-elements, standard $C^{1}$-elements, and special elements with built-in crack-tip functions, are considered. A proper quadrature rule is selected to efficiently and accurately evaluate both nearly and weakly singular double line integrals over pairs of elements resulting from the discretization and the solution of a dense system of linear algebraic equations is obtained using an efficient indirect solver. The rate of convergence of finite element solutions is fully investigated and such information is then used to conclude the influence of the residual surface tension on the behavior of the near-tip field.
\end{abstract}

Keywords: Gurtin-Murdoch surface elasticity, Integro-differential equations, Nano-sized cracks, Near-tip fields, Residual surface tension

\section{Introduction}

The framework of fracture mechanics based upon the classical continuum mechanics has been well established and commonly used in simulations of pre-existing cracks and flaws in a macro-scale and predicted results have been found in good agreement with experimental observations. However, this classical continuum-based theory, when applied directly to problems of nano-sized cracks, has failed to capture the actual physical phenomena due to the incapability of the underlying governing physics to mimic various inherent features such as the size-dependency, surface energy effects, non-locality, etc. However, due to their positive features such as the mathematical simplicity of governing physics and low requirement of computational resources in comparison with available discrete-based schemes (e.g., atomistic and molecular dynamics simulations), continuumbased theories and related techniques with the proper integration of intrinsic nano-scale influences (e.g., surface energy effect) to enhance the underlying governing physics have been continuously proposed. Gurtin-Murdoch surface elasticity theory (e.g., [1-2]) is, adopted over the past decades, to explore the effect of the material surface and size- 
dependent characteristics of mechanical properties of solids at the nano-scale. For instance, the model has been extensively used to study both static and dynamic mechanical behaviors of nano-wires and nano-plates [3-8], problems of nano-inhomogeneities [9], the surface energy effect on elastic properties of nano-sized wires, films, and particles [10], and elastic properties of both heterogeneous and homogeneous nano-structured materials (e.g., [11-18]).

However, applications of the surface elasticity theory to simulate nano-sized cracks have been quite limited as listed below. Wu [19] and Wu and Wang [20, 21] pointed out that the residual surface tension present at the crack face produces a pair of concentrated forces at the crack tips and this alters the crack-tip stress singularity from $1 / \sqrt{r}$ to $1 / r$. Wang et al. [22] applied Gurtin-Murdoch model to investigate finite rootradius cracks under mode-I and mode-III conditions. Results from their study confirmed the important role of surface stresses on the elastic field in the neighborhood of the crack tip as its radius of curvature falls within a nano-scale. The contribution of surface stresses on the dislocation emission was also studied by Fang et al. [23] for a crack with elliptical blunt tips under opening and shear modes. They concluded that as the crack size becomes comparable to the surface-material length scale, the surface stresses show the significant influence on the critical stress intensity factors. Later, $\mathrm{Fu}$ et al. [24, 25] applied finite element procedures to examine the surface stress effect on the near-front elastic field of blunt cracks subjected to mode-I and mode-II loading conditions. Wang and Li [26] also examined the role of the residual surface tension on the crack-tip field under mode-I loading condition and concluded that as the tip radius of curvature reduces from micro- to nana-scales, the stress field in the vicinity of the crack front deviates significantly from the case without the residual surface tension.

Models with a sharp crack tip were also extensively employed in literature to simulate nano-sized cracks. For instance, Oh et al. [27] resolved a Griffith crack problem by integrating the nano-scale effects. The crack opening displacement and stress distribution in a region surrounding the crack were studied using the information of the long-range intermolecular force from the discrete-based simulations. They also pointed out that the stress and the slope of the opened crack lines at the crack tip must be finite and, as a result, the sharp crack tip model should be used rather than the blunt one. Later, Kim et al. [28-30] investigated nano-sized cracks under mode-I, mode-II, and mode-III loading conditions and the effect of surface stresses via Gurtin-Murdoch model. A method of complex variable representation was adopted together with the physical-based assumption on the boundedness of the crack-tip stress. Sendova and Walton [31] further examined the influence of both curvature-independent and curvature-dependent residual surface tension on the elastic field of cracks in a whole plane. Results from their study concluded that the crack-tip stress singularity disappears when the curvature-dependent residual surface tension is considered and becomes logarithmic when the constant residual surface tension is treated. Such logarithmic crack-tip stress singularity was also concluded in the work of Kim et al. [32]. Later, Nan and Wang [33] explored the role of residual surface stresses (via Gurtin-Murdoch model) on both the stress intensity factors and the relative crack-face displacements. Obtained results confirmed that presence of surface stresses significantly alter the fracture response at the nano-scale; in particular, the residual surface tension was found affecting the crack-face displacement not only in the near-tip region but also at the whole crack line. Nan and Wang [34] extended their earlier work to investigate the influence of surface stresses on fracture responses of nano-scale cracks in a piezoelectric medium. They observed that the influence of residual surface stresses on the electromechanical responses and the electrical intensity factor was strongly affected by the 
electrical crack-face condition. The boundedness of the crack-tip strains and stresses for cracks under mode-I and mode-II loading conditions was also concluded in the recent work of Walton [35] if the curvature-dependent residual surface tension is used in the modeling. On basis of an extensive literature review, the influence of surface stresses and residual surface tension on the elastic field of nano-scale cracks, especially in the near-tip region, is still not well established and essentially requires further investigations. In particular, the conclusion on the boundedness and singularity of the crack-tip stress resulting from many studies are still inconsistent, questionable, and lacking of adequately mathematical proof.

In the present study, the near-tip elastic field of a finite-length, straight crack in a whole plane under mode-I loading is resolved by considering the influence of the residual surface tension. A surface elasticity theory by Gurtin and Murdoch [1,2] without the inplane stiffness is adopted in the formulation to describe the role of the residual surface tension. A numerical scheme based on Galerkin finite element method is utilized to efficiently solve the governing integro-differential equation for the unknown crackopening displacement. Results and findings from the present work should directly offer the fundamental insight of the near-tip elastic field when the surface effect is taken into account.

\section{Problem Formulation}

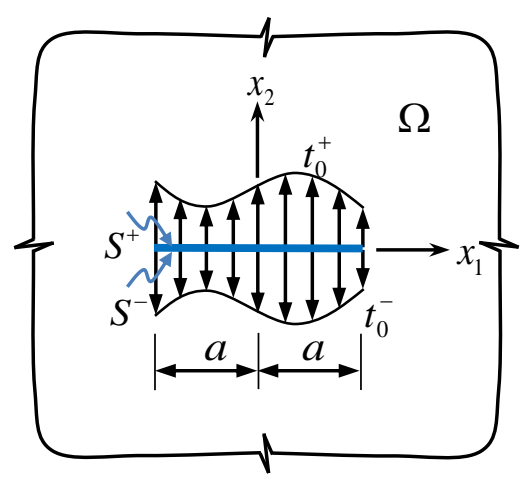

Figure 1. A whole-plane medium containing a straight crack of length $2 a$ under arbitrarily distributed normal traction

Consider a whole-plane, elastic medium $\Omega$ containing a straight crack of a length $2 a$ as illustrated in Figure 1. The crack, in the reference or undeformed state, possesses sharp crack tips and is represented by two coincident straight lines, termed the upper-crack line $S^{+}$and the lower-crack line $S^{-}$. Note that besides the geometric coincidence of $S^{+}$and $S^{-}$, unit normal vectors directing outward from the body at any coincident points of both crack lines are opposite (i.e., $\boldsymbol{n}^{+}=-\boldsymbol{n}^{-}$). The bulk material constituting the medium is assumed homogenous, isotropic, and linearly elastic with its properties completely described by the elastic shear modulus $\mu$ and Poisson's ratio $v$. The material layer on each crack line possesses the zero in-plane modulus and the constant residual surface tension $\tau^{s}$. The medium is loaded by arbitrarily distributed, self-equilibrated, normal tractions $t_{0}^{+}$on $S^{+}$and $t_{0}^{-}$on $S^{-}$(i.e., $t_{0}^{+}=-t_{0}^{-}$) and, in the present study, the body force is assumed negligible. For convenience in further reference, a planar Cartesian coordinate system $\left\{x_{1}, x_{2}\right\}$ is introduced such that its origin is at the center of the two coincident crack lines and the $x_{1}$ - and $x_{2}$-axes direct along and perpendicular to the crack lines, respectively, as indicated in Figure 1. 


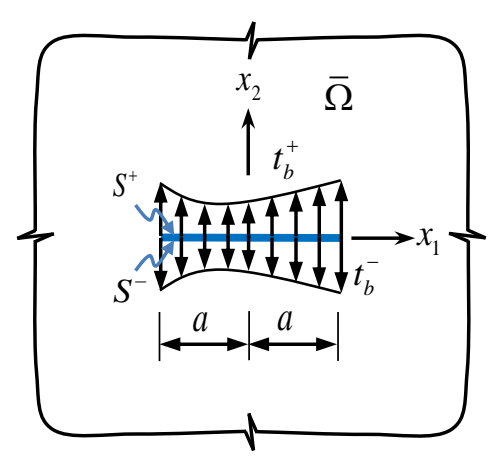

(a)

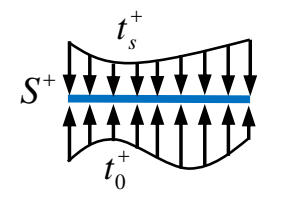

(b)

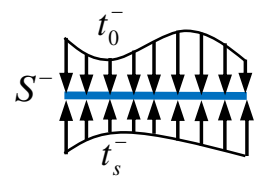

(c)

Figure 2. Schematics of (a) bulk cracked medium under unknown tractions $t_{b}^{+}$and $t_{b}^{-}$, (b) zero-thickness, two-sided material line $S^{+}$under known traction $t_{0}^{+}$and unknown traction $t_{s}^{+}$, and (c) zero-thickness, two-sided material line $S^{-}$under known traction $t_{0}^{-}$and unknown traction $t_{s}^{-}$

To establish the boundary value problem associated with the cracked body $\Omega$ described above, the medium is divided into three different parts: (i) a zero-thickness, twosided material line $S^{+}$peeled from the top crack line (see Figure 2(b)), (ii) a zerothickness, two-sided material line $S^{-}$peeled from the bottom crack line (see Figure 2(c)), (iii) the remaining bulk cracked medium $\bar{\Omega}$ (see Figure 2(a)). Since the thickness of the two material lines is zero, the geometry of the bulk cracked medium is clearly the same as that of the original cracked body. The key difference between $\bar{\Omega}$ and $\Omega$ is that the former is homogeneous but the latter is not. Resulting from the domain decomposition, the material line $S^{+}$is subjected to the known normal traction $t_{0}^{+}$and the unknown normal traction $t_{s}^{+}$exerted by the bulk part; the material line $S^{-}$is subjected to the known normal traction $t_{0}^{-}$and the unknown normal traction $t_{s}^{-}$exerted by the bulk part; and the bulk cracked medium is subjected to the unknown normal tractions $t_{b}^{+}$and $t_{b}^{-}$on the peeled cracked lines $S^{+}$and $S^{-}$, respectively. Note that the tractions induced at the interface between the bulk cracked medium and the two material lines have only the component normal to the crack lines since the material lines possess no in-plane modulus (this issue becomes more apparent in the development of the governing equation of the two material lines using Gurtin-Murdoch model). Since the two material lines are perfectly attached to the bulk cracked medium in the original cracked body, the traction and displacement developed along the material interface must be continuous, i.e.,

$$
\begin{gathered}
u_{\alpha}^{+b}\left(x_{1}, x_{2}=0\right)=u_{\alpha}^{+s}\left(x_{1}\right) ; u_{\alpha}^{-b}\left(x_{1}, x_{2}=0\right)=u_{\alpha}^{-s}\left(x_{1}\right) \quad \forall x_{1} \in[-a, a] \\
t_{b}^{+}\left(x_{1}\right)=-t_{s}^{+}\left(x_{1}\right) ; t_{b}^{-}\left(x_{1}\right)=-t_{s}^{-}\left(x_{1}\right) \quad \forall x_{1} \in[-a, a]
\end{gathered}
$$

where $u_{\alpha}^{+b}$ and $u_{\alpha}^{-b}$ are displacement components at $S^{+}$and $S^{-}$of the bulk cracked medium, respectively, and $u_{\alpha}^{+s}$ and $u_{\alpha}^{-s}$ are displacement components of the material lines $S^{+}$and $S^{-}$, respectively. Here and in what follows, superscripts or subscripts " $s$ " and " $b$ " are employed to designate quantities associated with the material lines and the bulk part, respectively. From the symmetry of the normal traction induced at the interface of the bulk 
cracked medium and each material line, it is apparent that such traction is self-equilibrated in the sense that $t_{b}^{+}+t_{b}^{-}=0$ and $t_{s}^{+}+t_{s}^{-}=0$.

The bulk cracked medium is mathematically modeled by a continuum theory of linear elasticity. For a two-dimensional medium made of a homogeneous, isotropic, linear elastic material, basic field equations, in the absence of the body force, are given by

$$
\begin{gathered}
\sigma_{\beta \alpha, \beta}^{b}=0 \\
\sigma_{11}^{b}=\mu \frac{\kappa+1}{\kappa-1} \varepsilon_{11}^{b}+\mu \frac{3-\kappa}{\kappa-1} \varepsilon_{22}^{b} ; \quad \sigma_{22}^{b}=\mu \frac{3-\kappa}{\kappa-1} \varepsilon_{11}^{b}+\mu \frac{\kappa+1}{\kappa-1} \varepsilon_{22}^{b} ; \sigma_{12}^{b}=2 \mu \varepsilon_{12}^{b} \\
\varepsilon_{\alpha \beta}^{b}=\frac{1}{2}\left(u_{\alpha, \beta}^{b}+u_{\beta, \alpha}^{b}\right)
\end{gathered}
$$

where $\sigma_{\beta \alpha}^{b}, \varepsilon_{\alpha \beta}^{b}$, and $u_{\alpha}^{b}$ denote components of the stress tensor, strain tensor and displacement vector of the bulk, respectively; $\kappa$ is a material constant given by $\kappa=3-4 v$ for the plane-strain condition and $\kappa=(3-v) /(1+v)$ for the plane-stress condition; and a standard indicial notation for the two-dimensional case applies

By recalling results reported by [36] for a semi-infinite, straight, dislocation embedded in a homogeneous, linear elastic, isotropic whole plane and the crack representation in terms of continuous dislocations (e.g., [37]), the relationship between the relative crack-face displacement of the bulk cracked medium shown in Figure 2(a) and the unknown normal tractions acting on both crack lines is given explicitly by

$$
t_{b}^{+}\left(x_{1}\right)=-t_{b}^{-}\left(x_{1}\right)=\int_{-a}^{a} \mathscr{K}\left(x_{1}, \xi\right) \frac{d \Delta u_{2}^{b}}{d \xi} d \xi \quad \forall x_{1} \in(-a, a)
$$

where $\Delta u_{2}^{b} \equiv u_{2}^{+b}-u_{2}^{-b}$ denotes the crack opening displacement of the bulk cracked medium and $\mathscr{K}\left(x_{1}, \xi\right)$ is a kernel defined by

$$
\mathscr{K}\left(x_{1}, \xi\right)=\frac{2 \mu}{\pi(\kappa+1)} \frac{1}{x_{1}-\xi}
$$

It is remarked that the kernel $\mathscr{K}\left(x_{1}, \xi\right)$ is singular at $x_{1}=\xi$ of $\mathcal{O}(r)$ with $r=\left|x_{1}-\xi\right|$ and the singular integral on the right hand side of (6) is of Cauchy-type. Note also that the crack opening displacement must satisfy the closure condition at the crack-tip, i.e., $\Delta u_{2}^{b}(-1)=\Delta u_{2}^{b}(1)=0$. Once the unknown crack opening displacement $\Delta u_{2}^{b}$ is solved, the displacements and stresses at any point $\boldsymbol{x}=\left(x_{1}, x_{2}\right)$ within the bulk cracked medium can be obtained from the following integral relations

$$
u_{\alpha}^{b}(\boldsymbol{x})=\int_{-a}^{a} \boldsymbol{u}_{\alpha}\left(x_{1}-\xi, x_{2}\right) \frac{d \Delta u_{2}^{b}}{d \xi} d \xi ; \quad \sigma_{\alpha \beta}^{b}(\boldsymbol{x})=\int_{-a}^{a} S_{\alpha \beta}\left(x_{1}-\xi, x_{2}\right) \frac{d \Delta u_{2}^{b}}{d \xi} d \xi
$$

where the kernels $\boldsymbol{u}_{\alpha}\left(x_{1}-\xi, x_{2}\right)$ and $\boldsymbol{S}_{\alpha \beta}\left(x_{1}-\xi, x_{2}\right)$ are defined by 


$$
\begin{gathered}
u_{1}(\bar{r}, \bar{\theta})=-\frac{1}{2 \pi(\kappa+1)}\{(\kappa-1) \ln \bar{r}-\cos 2 \bar{\theta}\}, u_{2}(\bar{r}, \bar{\theta})=-\frac{1}{2 \pi(\kappa+1)}\{(\kappa+1) \bar{\theta}-\sin 2 \bar{\theta}\} \\
S_{11}(\bar{r}, \bar{\theta})=-\frac{2 \mu}{\pi(\kappa+1)} \frac{\cos \bar{\theta} \cos 2 \bar{\theta}}{\bar{r}}, S_{12}(\bar{r}, \bar{\theta})=-\frac{2 \mu}{\pi(\kappa+1)} \frac{\sin \bar{\theta} \cos 2 \bar{\theta}}{\bar{r}} \\
S_{22}(\bar{r}, \bar{\theta})=-\frac{2 \mu}{\pi(\kappa+1)} \frac{\cos \bar{\theta}(2-\cos 2 \bar{\theta})}{\bar{r}}
\end{gathered}
$$

in which $\bar{r}=\sqrt{\left(x_{1}-\xi\right)^{2}+x_{2}^{2}}$ and $\bar{\theta}=\tan ^{-1}\left(x_{2} /\left(x_{1}-\xi\right)\right) \in[0,2 \pi]$.

Physical responses of a zero-thickness material line are mathematically modeled by Gurtin-Murdoch surface elasticity theory (e.g., [1-2]). In the absence of the in-plane modulus and for a straight material line located along the $x_{1}$-axis, the equilibrium equation and the constitutive law are given by

$$
\frac{d \sigma^{s}}{d x_{1}}+b^{s}=0 ; \quad \sigma^{s}=\tau^{s} \frac{d u_{2}^{s}}{d x_{1}}
$$

where $\sigma^{s}$ is the apparent out-of-plane shear stress within the material line induced from the membrane action and $b^{s}$ denotes the distributed force acting to the material line. Combining the two equations from (11) yields

$$
\frac{d}{d x_{1}}\left(\tau^{s} \frac{d u_{2}^{s}}{d x_{1}}\right)+b^{s}=0
$$

By applying (12) to the material line $S^{+}$shown in Figure 2(b) with $u_{2}^{s}=u_{2}^{+s}$ and $b^{s}=t_{0}^{+}+t_{s}^{+}$and the material line $S^{-}$shown in Figure 2(c) with $u_{2}^{s}=u_{2}^{-s}$ and $b^{s}=t_{0}^{-}+t_{s}^{-}$, and then performing the linear combination of the obtained results, it gives rise to

$$
\frac{d}{d x_{1}}\left(\tau^{s} \frac{d \Delta u_{2}^{s}}{d x_{1}}\right)+2 t_{0}^{+}+2 t_{s}^{+}=0
$$

where $\Delta u_{2}^{s} \equiv u_{2}^{+s}-u_{2}^{-s}$ denotes the crack opening displacement. By applying the conditions (1) - (2), the boundary integral equation for the bulk cracked medium (6) and the governing differential equation for the two material lines (13) can be properly combined into a governing integro-differential equation for the original cracked body:

$$
\frac{1}{2} \frac{d}{d x_{1}}\left(\tau^{s} \frac{d \Delta u_{2}^{b}}{d x_{1}}\right)+t_{0}^{+}=\int_{-a}^{a} \mathscr{K}\left(x_{1}, \xi\right) \frac{d \Delta u_{2}^{b}}{d \xi} d \xi \forall x_{1} \in(-a, a)
$$

By defining following non-dimensional parameters $\Lambda=\tau^{s o} / \mu, \bar{a}=a / \Lambda, \bar{\tau}^{s}=\tau^{s} / \tau^{s o}$, $\bar{x}=x_{1} / a, \bar{\xi}=\xi / a, \overline{t_{0}}=t_{0}^{+} / \mu, \Delta \bar{u}=\Delta u_{2}^{b} / a$ and $\bar{\kappa}=4 / \pi(\kappa+1)$ where $\tau^{s o}$ is the reference value of the residual surface tension, the integro-differential equation (14) becomes 


$$
\frac{d}{d \bar{x}}\left(\frac{\bar{\tau}^{s}}{\bar{a}} \frac{d \Delta \bar{u}}{d \bar{x}}\right)+2 \bar{t}_{0}=-\int_{-1}^{1} \overline{\mathscr{K}}(\bar{x}, \bar{\xi}) \frac{d \Delta \bar{u}}{d \bar{\xi}} d \bar{\xi} \quad \forall \bar{x} \in(-1,1)
$$

where $\overline{\mathscr{K}}(\bar{x}, \bar{\xi})$ is given by

$$
\overline{\mathscr{K}}(\bar{x}, \bar{\xi})=\bar{\kappa} /(\bar{\xi}-\bar{x})
$$

To construct the weak statement of Eq. (15), a weighted residual technique is utilized as follows. By first multiplying (15) with a sufficiently smooth weight function $w=w(\bar{x})$ satisfying the closure conditions $w(-1)=w(1)=0$, then integrating the obtained result along the whole crack line, and finally performing the integration by parts together with enforcing the closure conditions of the weight function $w$, it leads to

$$
-\int_{-1}^{1} \frac{\bar{\tau}^{s}}{\bar{a}} \frac{d w}{d \bar{x}} \frac{d \Delta \bar{u}}{d \bar{x}} d \bar{x}+2 \int_{-1}^{1} w \overline{t_{0}} d \bar{x}=-\int_{-1}^{1} w \int_{-1}^{1} \overline{\mathscr{K}}(\bar{x}, \bar{\xi}) \frac{d \Delta \bar{u}}{d \bar{\xi}} d \bar{\xi} d \bar{x}
$$

To further reduce the singularity present in the double line integral of (26), the strongly singular kernel $\overline{\mathscr{K}}(\bar{x}, \bar{\xi})$ is first represented by

$$
\overline{\mathscr{K}}(\bar{x}, \bar{\xi})=\frac{\partial}{\partial \bar{x}} \mathscr{W}(\bar{x}, \bar{\xi}), \mathscr{W}(\bar{x}, \bar{\xi})=-\bar{\kappa} \ln |\bar{\xi}-\bar{x}|
$$

It is remarked that the kernel $\mathscr{W}(\bar{x}, \bar{\xi})$ is obviously singular at $\bar{\xi}=\bar{x}$ of $\mathcal{O}(\ln r)$ with $r=|\bar{\xi}-\bar{x}|$. By applying the representation (18) to (17) and then performing the integration by parts of the double line integral, it finally yields

$$
\int_{-1}^{1} \frac{d w}{d \bar{x}} \int_{-1}^{1} \mathscr{W}(\bar{x}, \bar{\xi}) \frac{d \Delta \bar{u}}{d \bar{\xi}} d \bar{\xi} d \bar{x}+\int_{-1}^{1} \frac{\bar{\tau}^{s}}{\bar{a}} \frac{d w}{d \bar{x}} \frac{d \Delta \bar{u}}{d \bar{x}} d \bar{x}=2 \int_{-1}^{1} w \overline{t_{0}} d \bar{x}
$$

The weak-form equation (19) is apparently in a symmetric form (i.e., $\Delta \bar{u}$ and $w$ contained in the two integrals on the left hand side of (19) can be interchanged without changing their meaning) and the double integral contains only a weakly singular kernel allowing the interpretation in the sense of Riemann sum.

\section{Numerical Implementations}

Following Galerkin approximation procedure, the crack-opening displacement and the weight function over the normalized interval $[-1,1]$ can be discretized by

$$
\Delta \bar{u}^{h}(\bar{x})=\sum_{i=1}^{N} \Delta \bar{u}_{i} \phi_{i}(\bar{x}) ; \quad w^{h}(\bar{x})=\sum_{i=1}^{N} w_{i} \phi_{i}(\bar{x})
$$

where $\phi_{i}(\bar{x})$ denote the basic functions satisfying the closure conditions $\phi_{i}(-1)=\phi_{i}(1)=0$; $\Delta \bar{u}_{i}$ are unknown degrees of freedom; $w_{i}$ are arbitrary constants; and $N$ is the number of degrees of freedom resulting from the discretization. By substituting the approximations (20) into the weak-form equation (19) and then using the arbitrariness of the weight function $w^{h}$, it gives rise to a system of linear algebraic equations 


$$
\sum_{j=1}^{N} K_{i j} \Delta u_{j}=F_{i}, \quad \forall i \in\{1,2,3, \ldots, N\}
$$

where entries of the coefficient matrix and the load vector are given by

$$
\begin{gathered}
K_{i j}=\hat{K}_{i j}+\bar{K}_{i j} ; \quad \hat{K}_{i j}=\int_{-1}^{1} \frac{d \phi_{i}}{d \bar{x}} \int_{-1}^{1} \mathscr{W}(\bar{x}, \bar{\xi}) \frac{d \phi_{j}}{d \bar{\xi}} d \bar{\xi} d \bar{x} ; \quad \bar{K}_{i j}=\int_{-1}^{1} \frac{\bar{\tau}^{s}}{\bar{a}} \frac{d \phi_{i}}{d \bar{x}} \frac{d \phi_{j}}{d \bar{x}} d \bar{x} \\
F_{i}=2 \int_{-1}^{1} \phi_{i} \bar{t}_{0} d \bar{x}
\end{gathered}
$$

It is apparent that the coefficient matrix $\boldsymbol{K}$ is essentially symmetric. A finite element technique is employed to systematically construct the basis functions $\phi_{i}(\bar{x})$ used in the discretization. First, the domain $[-1,1]$ is discretized into a finite element mesh containing $m$ elements of equal length $h$ and $n$ nodes. For any generic element $\Omega_{e}=\left[\bar{x}_{e}, \bar{x}_{e+1}\right]$ containing $n_{e}$ nodes, the element shape functions, denoted by $\psi_{I}^{e}\left(\bar{x}^{e}\right), I=1,2, \ldots, n_{e}$ where the uppercase index $I$ is used to indicate the local labeling of nodes in the element and $\bar{x}^{e}$ is the local coordinate defined specifically for each element, are constructed and used to form the nodal basis functions at any global node $i, \phi_{i}(\bar{x})$, by a simple patching algorithm. In the present study, several types of elements including standard $C^{0}$-linear elements (Type-1), $C^{0}$-quadratic elements (Type-2), $C^{0}$-cubic elements (Type-3) and $C^{1}$ hermite elements (Type-4), defined on a master element $\eta \in[-1,1]$, and special $C^{0}$ elements with a built-in function to capture the crack-tip singularity are considered. By using the finite element procedure to construct the nodal basis functions $\phi_{i}(\bar{x})$, the formula (22) and (23) become

$$
K_{i j}=\sum_{g=1}^{m} \sum_{e=1}^{m} \hat{k}_{I J}^{e g}+\sum_{e=1}^{m} \bar{k}_{I J}^{e} ; \quad F_{i}=\sum_{e=1}^{m} f_{I}^{e}
$$

where the local and global labeling of nodes has the one-to-one correspondence $i=i(I)$; $\hat{k}_{I J}^{e g}$ denotes the contribution of $\hat{K}_{i j}$ from a pair of elements $\Omega_{e}$ and $\Omega_{g} ; \bar{k}_{I J}^{e}$ denotes the contribution of $\bar{K}_{i j}$ from the element $\Omega_{e} ; f_{I}^{e}$ denotes the contribution of $F_{i}$ from the element $\Omega_{e}$; and the summation appearing in (24) can be achieved in an efficient manner using a direct assembly scheme. Entries of the element matrices and load vectors $\hat{k}_{I J}^{e g}, \bar{k}_{I J}^{e}$ and $f_{I}^{e}$ can be expressed explicitly in terms of the element shape functions $\psi_{I}^{e}\left(\bar{x}^{e}\right)$ as

$$
\hat{k}_{I J}^{e g}=\int_{\Omega_{e}} \frac{d \psi_{I}^{e}}{d \bar{x}^{e}} \int_{\Omega_{g}} \mathscr{W}(\bar{x}, \bar{\xi}) \frac{d \psi_{J}^{g}}{d \bar{\xi}^{g}} d \bar{\xi}^{g} d \bar{x}^{e} ; \quad \bar{k}_{I J}^{e}=\int_{\Omega_{e}} \frac{\bar{\tau}^{s}}{\bar{a}} \frac{d \psi_{I}^{e}}{d \bar{x}^{e}} \frac{d \psi_{J}^{e}}{d \bar{x}^{e}} d \bar{x}^{e} ; \quad f_{I}^{e}=2 \int_{\Omega_{e}} \psi_{I}^{e} \bar{t}_{0} d \bar{x}^{e}
$$

To construct special $C^{0}$ crack-tip elements for capturing the near-tip field, results from [31] are used. In that work, it is concluded that the crack-opening displacement has a finite slope at the crack tip and this, as a result, renders the stress field singular at the crack-tip of 
order $\ln r$ where $r$ denotes the normalized distance from the crack-tip. Such argument implies that the near-tip crack-opening displacement should take the following form

$$
\Delta \bar{u}=K r^{2} \ln r+C r+h(r)
$$

where $K$ and $C$ are unknown constants and $h=h(r)$ is a function satisfying $h(0)=0$ and the following condition

$$
\lim _{r \rightarrow 0}\left(\frac{h(r)}{r^{2} \ln r}\right)=0
$$

The second term of (26) is needed to ensure the finite slope of $\Delta \bar{u}$ at the crack tip whereas the condition (27) simply indicates that the singularity induced from the derivatives of the function $h=h(r)$ is weaker than that of the function $r^{2} \ln r$ and the first term of (26) dominates the near-tip field. Special crack-tip elements with their element shape functions being properly enriched to contain the dominant terms given by (26) are developed and then used to capture the near-tip crack-opening displacement. If the argument set forth by [31] is right, the finite element solutions obtained by using special crack-tip elements at both crack tips will yield a finite value of $K$ as the mesh is refined. However, if the cracktip singularity is weaker or stronger than that generated by the function $r^{2} \ln r$, the constant $K$ will converge to zero or diverge to infinity as the mesh is refined.

To construct a special $C^{0}$ crack-tip element of length $l_{e}$ containing two nodes, the element shape functions are assumed in the following form

$$
\psi_{I}^{e}(r)=C_{1 I} r^{2} \ln r+C_{2 I} r, \quad I=1,2
$$

where the constants $C_{1 I}$ and $C_{2 I}$ can be obtained from the conditions $\psi_{1}^{e^{\prime}}(0)=1$, $\psi_{1}^{e}\left(l_{e}\right)=0, \psi_{2}^{e^{\prime}}(0)=0$ and $\psi_{2}^{e}\left(l_{e}\right)=1$. The final form of special element shape functions for this particular element, expressed in terms of a master coordinate $\eta \in[-1,1]$, is given by

$$
\psi_{1}^{e}(\eta)=l_{e} \bar{\eta}\left[1-\bar{\eta} \ln \left(\bar{\eta} l_{e}\right) / \ln l_{e}\right] ; \psi_{2}^{e}(\eta)=\bar{\eta}^{2} \ln \left(\bar{\eta} l_{e}\right) / \ln l_{e}
$$

where $\bar{\eta}=(1+\eta) / 2$. Similarly, a special $C^{0}$ crack-tip element of length $l_{e}$ containing three nodes (Type-5) can be obtained by assuming shape functions of the following form

$$
\psi_{I}^{e}(r)=C_{1 I} r^{2} \ln r+C_{2 I} r+C_{3 I} r^{2}, \quad I=1,2,3
$$

where, again, the unknown constants $C_{1 I}, C_{2 I}$ and $C_{3 I}$ can be obtained from the following conditions $\quad \psi_{1}^{e^{\prime}}(0)=1, \quad \psi_{1}^{e}\left(l_{e} / 2\right)=0, \quad \psi_{1}^{e}\left(l_{e}\right)=0, \quad \psi_{2}^{e^{\prime}}(0)=0, \quad \psi_{2}^{e}\left(l_{e} / 2\right)=1, \quad \psi_{2}^{e}\left(l_{e}\right)=0$, $\psi_{3}^{e^{\prime}}(0)=0, \psi_{3}^{e}\left(l_{e} / 2\right)=0$, and $\psi_{3}^{e}\left(l_{e}\right)=1$. The final form of special element shape functions (30), expressed in terms of the master coordinate $\eta \in[-1,1]$, is given by

$$
\psi_{1}^{e}(\eta)=l_{e} \bar{\eta}[1+\bar{\eta} \ln (\bar{\eta} / 2) / \ln 2] ; \quad \psi_{2}^{e}(\eta)=-4 \bar{\eta}^{2} \ln \bar{\eta} / \ln 2 ; \quad \psi_{3}^{e}(\eta)=\bar{\eta}^{2} \ln (2 \bar{\eta}) / \ln 2
$$


Finally, a special $C^{0}$ crack-tip element of length $l_{e}$ containing four nodes (Type-6) can be constructed by assuming its shape functions of the following form

$$
\psi_{I}^{e}(r)=C_{1 I} r^{2} \ln r+C_{2 I} r+C_{3 I} r^{2}+C_{4 I} r^{3}, \quad I=1,2,3,4
$$

where all unknown constants $C_{1 I}, C_{2 I}, C_{3 I}$ and $C_{4 I}$ can be obtained from following conditions $\quad \psi_{1}^{e^{\prime}}(0)=1, \quad \psi_{1}^{e}\left(l_{e} / 3\right)=0, \quad \psi_{1}^{e}\left(2 l_{e} / 3\right)=0, \quad \psi_{1}^{e}\left(l_{e}\right)=0, \quad \psi_{2}^{e^{\prime}}(0)=0, \quad \psi_{2}^{e}\left(l_{e} / 3\right)=1$, $\psi_{2}^{e}\left(2 l_{e} / 3\right)=0, \quad \psi_{2}^{e}\left(l_{e}\right)=0, \quad \psi_{3}^{e^{\prime}}(0)=0, \quad \psi_{3}^{e}\left(l_{e} / 3\right)=0, \quad \psi_{3}^{e}\left(2 l_{e} / 3\right)=1, \quad \psi_{3}^{e}\left(l_{e}\right)=0, \quad \psi_{4}^{e^{\prime}}(0)=0$, $\psi_{4}^{e}\left(l_{e} / 3\right)=0, \quad \psi_{4}^{e}\left(2 l_{e} / 3\right)=0$ and $\psi_{4}^{e}\left(l_{e}\right)=1$. The final form of special element shape functions (32), expressed in terms of the master coordinate $\eta \in[-1,1]$, is given by

$$
\begin{aligned}
& \psi_{1}^{e}(\eta)=l_{e} \bar{\eta}\left\{3 \ln (27 / 16) \bar{\eta}^{2}-[11 \ln 3+2 \ln (\bar{\eta} / 256)] \bar{\eta}+2 \ln (3 / 4)\right\} / 2 \ln (3 / 4) \\
& \psi_{2}^{e}(\eta)=9 \bar{\eta}^{2}[\ln (27 \bar{\eta} / 8)+\ln (8 / 27) \bar{\eta}] / \ln (3 / 4) \\
& \psi_{3}^{e}(\eta)=-9 \bar{\eta}^{2}\left[\ln \left(27 \bar{\eta}^{2}\right)-\ln (27) \bar{\eta}\right] / 4 \ln (3 / 4) \\
& \psi_{4}^{e}(\eta)=\bar{\eta}^{2}[\ln (6 \bar{\eta})-\ln (8) \bar{\eta}] / \ln (3 / 4)
\end{aligned}
$$

where $r=\bar{\eta} l_{e}$. It is worth noting that the special shape functions developed above are applied only to the element containing the left crack-tip; one containing the right crack-tip can be readily constructed in the same manner.

It is evident from (25) that the entries $\bar{k}_{I J}^{e}$ and $f_{I}^{e}$ involve only regular integrals and they can be evaluated efficiently by standard Gaussian quadrature. On the contrary, the entries $\hat{k}_{I J}^{e g}$ involve a double line integral whose integrand can be regular, nearly singular, or weakly singular depending on a pair of elements involved. If the pair of elements $\left\{\Omega_{e}, \Omega_{g}\right\}$ is relatively remote in comparison with the element size, the kernel $\mathscr{W}(\bar{x}, \bar{\xi})$ is well-behaved and $\hat{k}_{I J}^{e g}$ can be integrated efficiently by standard Gaussian quadrature. If elements $\left\{\Omega_{e}, \Omega_{g}\right\}$ in the pair are adjacent or identical, the integrand can be either nearly singular or weakly singular, and the corresponding double line integral cannot be efficiently evaluated by Gaussian quadrature. In the present study, quadrature rules based on special coordinate transformations proposed by [38] are adopted to treat both the logarithmic singularity and rapid variation of the integrand.

To investigate the accuracy of the approximation for any finite element mesh, an error function, denoted by $e=e(\bar{x})$ for $\bar{x} \in[-1,1]$, is first defined by

$$
e(\bar{x})=\Delta \bar{u}^{r e f}(\bar{x})-\Delta \bar{u}^{h}(\bar{x})
$$

where $\Delta \bar{u}^{\text {ref }}(\bar{x})$ denotes the reference solution of the crack-opening displacement obtained from a sufficiently fine mesh to ensure its convergence and $\Delta \bar{u}^{h}(\bar{x})$ denotes the finite element solution of the crack-opening displacement for any given mesh. Next, the following norm is employed to measure the magnitude of the error function:

$$
\|e\|=\sqrt{\int_{\Omega} e^{2}(\bar{x})+\left[e^{\prime}(\bar{x})\right]^{2} d \bar{x}}
$$


Note that the evaluation of the above integral can be achieved efficiently, in an elementby-element fashion, over the finite element mesh. Finally, the relative error $\hat{E}$ is defined by normalizing the error norm (35) by the same norm of the reference solution, i.e.,

$$
\hat{E}=\|e\| /\left\|\Delta \bar{u}^{r e f}\right\|
$$

The rate of convergence can be estimated from the norm of the error function $\|e\|$ from the following relation

$$
\lim _{h_{e} \rightarrow 0}\left(\left\|e_{h_{e}}\right\| /\left\|e_{h_{e} / 2}\right\|\right)=2^{p}
$$

where $\left\|e_{h_{e}}\right\|$ and $\left\|e_{h_{e} / 2}\right\|$ are norms of the error function associated with the uniform meshes containing elements of the size $h_{e}$ and $h_{e} / 2$, respectively, and $p$ is a real number representing the rate of convergence of numerical solutions. It is worth noting that the rate of convergence depends primarily on the choice of basic functions used in the finite element approximation, the norm used to measure the error function, and the regularity of the true solution. If $p$ is positive then the error norm $\|e\|$ approaches zero as $h$ tends to zero. If the error norm $\|e\|$ approaches zero, an approximate solution is said to converge to the exact solution with respect to the norm $\|\cdot\|$.

\section{Numerical Results}

In the present study, a crack under a self-equilibrated, uniformly distributed normal traction is investigated. Material properties used in the simulations are taken from [39]; in particular, Poisson's ratio and Young modulus of the bulk medium and the residual surface tension of the two material layers are taken as $v=0.3, E=89.5 \mathrm{GPa}$ and $\tau^{s}=0.9108 \mathrm{~N} / \mathrm{m}$, respectively. The reference residual surface tension is taken from the work of [12] (i.e., $\tau^{s o}=1.3 \mathrm{~N} / \mathrm{m}$ ).

To verify the implemented numerical procedure, results of the crack-face displacement gradient at the crack tip are compared with the benchmark solution (obtained from [31]) in Table 1 and Table 2 for various types of elements and different levels of discretization. It is seen that computed crack-face displacement gradients at the right crack tip converge to a finite value and agree with the reference solution for all types of elements as the mesh is refined. Among all standard elements considered in the present study, results generated from elements of Type-3 and Type- 4 are comparable and converge to the benchmark solution faster than those obtained from elements of Type-2 and Type-1, respectively. It is also apparent that use of special elements (Type-5 and Type-6) at the crack tips along with standard elements (Type-2 and Type-3) for the remaining crack yields more accurate results than those generated by standard elements for the same level of discretization.

Since the exact solution is unknown a priori, the true error cannot be computed. It is remarked however that the finite element solutions can be improved and they finally converge to the exact solution when the number of elements is sufficiently large. Based on results obtained above, the combination of elements of Type- 3 and Type- 6 yields the best solution in comparison with other types of elements considered. As a result, a mesh containing totally 4096 elements (with 4094 elements of Type-3 and 2 special crack-tip elements of Type-6) is employed to generate the reference solution used for calculating the 
error function and the corresponding error norm. The log-log plot of the relative error $\hat{E}$ versus the size of elements used in the discretization is reported in Figure 3 for various types of elements. A slope of each plot in a region where the size of the elements is sufficiently small represents the rate of convergence $p$ in (37). It can be concluded from this set of results that the element of Type- 1 yields the lowest rate of convergence $(p \approx 1)$ whereas the rest yields about the same rate of convergence $(p \approx 1.5)$. This implies that increase in the degree of polynomials in the approximation does not affect the rate of convergence and this stems directly from the irregularity of the solution at the crack tips. In addition, use of special crack-tip elements to enhance the approximation of the localized near-tip field cannot improve the global rate of convergence of numerical solutions.

Table 1. Crack-face Displacement Gradient at the Right Crack Tip Obtained from Four Types of Standard Elements and Two Types of Special Crack-tip Elements

Results are reported for $\bar{t}_{0}=0.00266, \bar{\kappa}=0.475$ and $\bar{\tau}^{s} / \bar{a}=0.133$ and compared with that reported by [31].

\begin{tabular}{|c|c|c|c|c|c|c|}
\hline \multirow{2}{*}{$\begin{array}{c}\text { No. } \\
\text { Elements }\end{array}$} & \multicolumn{5}{|c|}{$\bar{u}^{\prime}(\bar{x}=1)$} \\
\cline { 2 - 7 } & Type-1 & Type-2 & Type-3 & Type-4 & $\begin{array}{c}\text { Type2 + } \\
\text { Type-5 }\end{array}$ & $\begin{array}{c}\text { Type3 + } \\
\text { Type-6 }\end{array}$ \\
\hline 2 & -0.0017 & -0.0036 & -0.0046 & -0.0044 & -0.0054 & -0.0063 \\
\hline 4 & -0.0026 & -0.0045 & -0.0055 & -0.0054 & -0.0062 & -0.0067 \\
\hline 8 & -0.0035 & -0.0054 & -0.0061 & -0.0061 & -0.0066 & $\mathbf{- 0 . 0 0 6 9}$ \\
\hline 16 & -0.0044 & -0.0060 & -0.0065 & -0.0065 & -0.0068 & $\mathbf{- 0 . 0 0 6 9}$ \\
\hline 32 & -0.0052 & -0.0064 & -0.0067 & -0.0067 & $\mathbf{- 0 . 0 0 6 9}$ & $\mathbf{- 0 . 0 0 6 9}$ \\
\hline 64 & -0.0058 & -0.0067 & -0.0068 & -0.0068 & $\mathbf{- 0 . 0 0 6 9}$ & $\mathbf{- 0 . 0 0 6 9}$ \\
\hline 128 & -0.0062 & -0.0068 & $\mathbf{- 0 . 0 0 6 9}$ & $\mathbf{- 0 . 0 0 6 9}$ & $\mathbf{- 0 . 0 0 6 9}$ & $\mathbf{- 0 . 0 0 6 9}$ \\
\hline 256 & -0.0065 & $\mathbf{- 0 . 0 0 6 9}$ & $\mathbf{- 0 . 0 0 6 9}$ & $\mathbf{- 0 . 0 0 6 9}$ & $\mathbf{- 0 . 0 0 6 9}$ & $\mathbf{- 0 . 0 0 6 9}$ \\
\hline 512 & -0.0067 & $\mathbf{- 0 . 0 0 6 9}$ & $\mathbf{- 0 . 0 0 6 9}$ & $\mathbf{- 0 . 0 0 6 9}$ & $\mathbf{- 0 . 0 0 6 9}$ & $\mathbf{- 0 . 0 0 6 9}$ \\
\hline 1024 & -0.0068 & $\mathbf{- 0 . 0 0 6 9}$ & $\mathbf{- 0 . 0 0 6 9}$ & $\mathbf{- 0 . 0 0 6 9}$ & $\mathbf{- 0 . 0 0 6 9}$ & $\mathbf{- 0 . 0 0 6 9}$ \\
\hline 2048 & $\mathbf{- 0 . 0 0 6 9}$ & $\mathbf{- 0 . 0 0 6 9}$ & $\mathbf{- 0 . 0 0 6 9}$ & $\mathbf{- 0 . 0 0 6 9}$ & $\mathbf{- 0 . 0 0 6 9}$ & $\mathbf{- 0 . 0 0 6 9}$ \\
\hline$[31]$ & \multicolumn{7}{|c|}{} \\
\hline
\end{tabular}

Table 2. Values of $K$ Extracted from Solutions Generated by Special Crack-tip Elements of Type-5 and Type-6

\begin{tabular}{|c|l|l|}
\hline \multirow{2}{*}{ No. Elements } & \multicolumn{2}{|c|}{$K$} \\
\cline { 2 - 3 } & Type-5 & Type-6 \\
\hline 2 & 0.320 & 0.473 \\
\hline 4 & 0.394 & 0.453 \\
\hline 8 & 0.422 & 0.434 \\
\hline 16 & 0.426 & 0.419 \\
\hline 32 & 0.421 & 0.407 \\
\hline 64 & 0.413 & 0.400 \\
\hline 128 & 0.406 & 0.396 \\
\hline 256 & 0.400 & 0.394 \\
\hline 512 & 0.397 & 0.393 \\
\hline 1024 & 0.394 & 0.393 \\
\hline 2048 & 0.393 & 0.393 \\
\hline
\end{tabular}




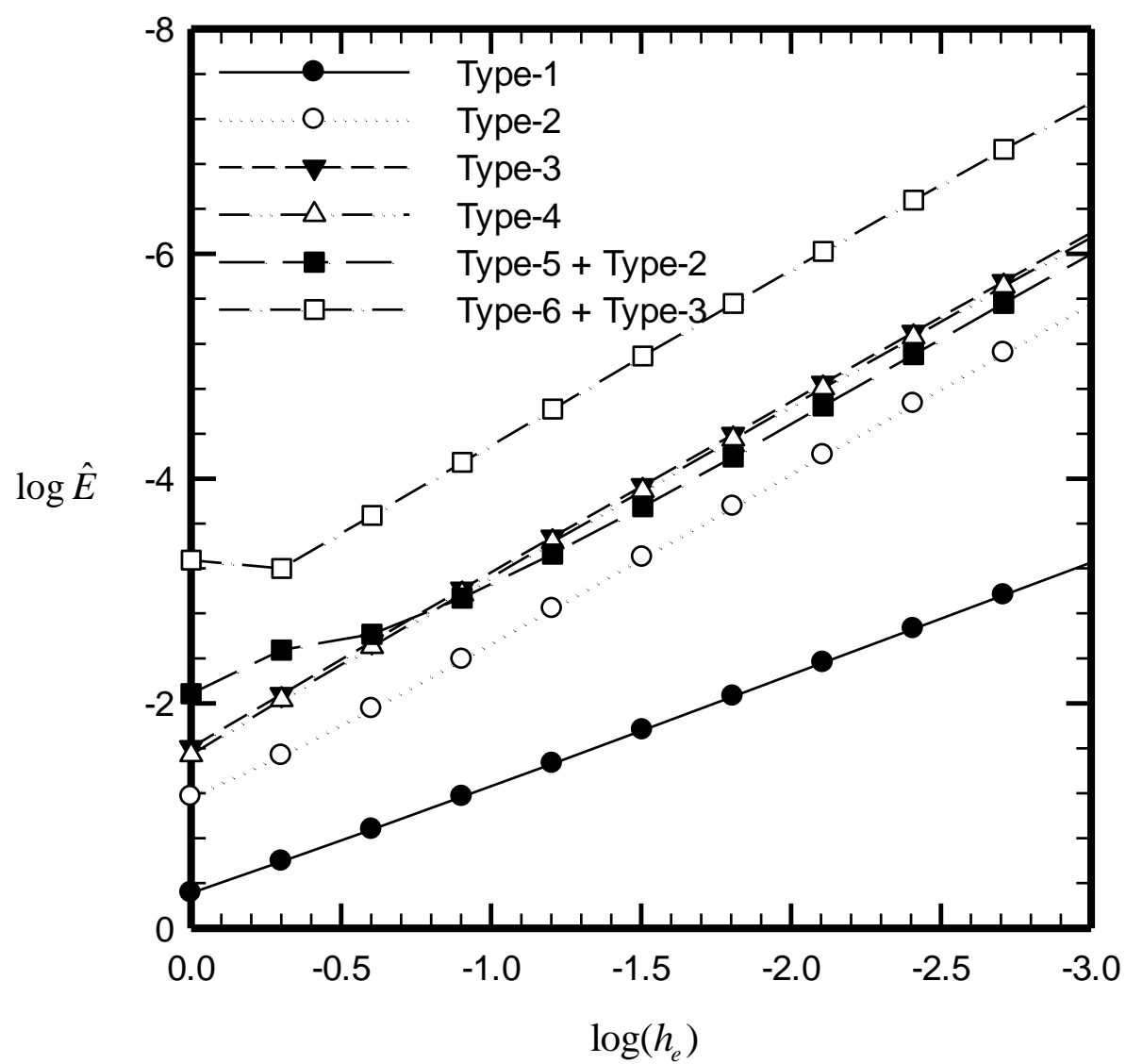

Figure 3. Log-log plots of relative error $\hat{E}$ versus element size $h_{e}$ for various types of elements. Results are simulated for $\bar{\tau}^{s} / \bar{a}=1$, and $\bar{t}_{o}=1$.

From the computed results, the coefficient $K$ in front of the dominant term $r^{2} \ln r$ of the near-tip crack opening displacement given by (26) is extracted and then reported in Table 2 when the two types of special crack-tip elements (i.e., Type-5 and Type-6) are employed to model the near-tip field. It is evident from these results that as the mesh is refined, the coefficient $K$ seems converge to a finite value (i.e., $K \approx 0.39$ ) for both types of special crack-tip elements. This finding should additionally confirm the argument concluded by [31]; specifically, the stress filed is singular at the crack tip of order $\ln r$ when the residual surface tension is incorporated in the mathematical model or, equivalently, the near-tip crack-opening displacement is dominated by $r^{2} \ln r$.

\section{Conclusions}

The convergence behavior of finite element solutions for the near-tip field of a finite straight crack in a two-dimensional, elastic, infinite medium under the pure mode-I loading condition and the surface effects has been explored. The classical theory of linear elasticity together with Gurtin-Murdoch surface elasticity has been employed to formulate a secondorder, integro-differential equation governing the relative crack-face displacement. The standard weighted residual technique has been applied to construct a weakly singular weakform statement. Galerkin strategy and the finite element approximation have been used to discretize the governing equation to obtain the approximate solution. Six types of element 
shape functions, generated by standard $C^{0}$ and $C^{1}$ elements, and special elements with built-in crack-tip functions have been employed to generate finite element basis functions. The standard Gaussian quadrature and logarithm Gaussian integration have been selected to efficiently and accurately evaluate both nearly singular and weakly singular double line integrals over pairs of elements resulting from the discretization and the solution of a dense system of linear algebraic equations has been obtained using an efficient indirect solver.

Results from a numerical study have shown that the rate of convergence of numerical solutions is controlled by the regularity of the solution at the crack tips and cannot be enhanced by increasing the degree of polynomials used in the approximation. Use of special crack-tip elements whose shape functions are properly enriched to capture the term $r^{2} \ln r$ has been found yielding better convergence for the crack-face displacement gradient at the crack tip but providing no improvement of the rate of convergence of numerical solutions over the crack. However, the coefficient in front of the term $r^{2} \ln r$ in the expansion of the near-tip crack-face displacement has been found converged to a finite real number as the mesh is refined. This therefore confirms the argument addressed in [31] that integration of the residual surface tension into the mathematic model weakens the crack-tip stress singularity from $r^{-1 / 2}$ in the classical theory to $\ln r$.

\section{Acknowledgements}

The authors gratefully acknowledge the support provided by Thailand Research Fund (Grant No. BRG5880017).

\section{References}

[1] M.E. Gurtin, and A.I. Murdoch, "A continuum theory of elastic material surfaces," Archive for Rational Mechanics and Analysis, Vol. 57, No. 4, pp. 291-323, 1975.

[2] M.E. Gurtin, and A.I. Murdoch, "Surface stress in solids," International Journal of Solids and Structures, Vol. 14, No. 6, pp. 431-440, 1978.

[3] G. Wang, and X. Feng, "Effects of surface elasticity and residual surface tension on the natural frequency of microbeams," Applied Physics Letters, Vol. 90, No. 23, pp. 231904-1-231904-3, 2007.

[4] G. Wang, and X. Feng, "Timoshenko beam model for buckling and vibration of nanowires with surface effects," Journal of Physics D: Applied Physics, Vol. 42, No. 15, pp.155411-1-155411-5, 2009.

[5] G. Wang, and F. Yang, "Postbuckling analysis of nanowires with surface effects," Journal of Applied Physics, Vol. 109, No. 6, pp. 063535-1-063535-4, 2011.

[6] K.F. Wang, and B.L. Wang, "Combining effects of surface energy and non-local elasticity on the buckling of nanoplates," Micro \& Nano Letters, Vol. 6, No. 11, pp. 941-943, 2011.

[7] K.F. Wang, and B.L. Wang, "Vibration of nanoscale plates with surface energy via nonlocal elasticity," Physica E: Low-dimensional Systems and Nanostructures, Vol. 44, No. 2, pp. 448-453, 2011.

[8] K.F. Wang, and B.L. Wang, "Effects of residual surface stress and surface elasticity on the nonlinear free vibration of nanoscale plates," Journal of Applied Physics, Vol. 112, No. 1, p. 013520, 2012.

[9] P. Sharma, S. Ganti, and N. Bhate, "Effect of surfaces on the size-dependent elastic state of nano-inhomogeneities," Applied Physics Letters, Vol. 82, No. 4, pp. 535$537,2003$. 
[10] R. Dingreville, J. Qu, and C. Mohammed, "Surface free energy and its effect on the elastic behavior of nano-sized particles, wires and films", Journal of the Mechanics and Physics of Solids, Vol. 53, No. 8, pp. 1827-1854, 2005.

[11] R.C. Cammarata, "Surface and interface stress effects on interfacial and nanostructured materials," Materials Science and Engineering: A, Vol. 237, No. 2, pp.180-184, 1997.

[12] P. Sharma, and S. Ganti, "Size-dependent Eshelby's tensor for embedded nanoinclusions incorporating surface/interface energies," Journal of Applied Mechanics, Vol. 71, No. 5, pp. 663-671, 2004.

[13] V.B. Shenoy, "Atomistic calculations of elastic properties of metallic fcc crystal surfaces," Physical Review B, Vol. 71, No. 9, pp. 094104-1, 2005.

[14] H. Duan, J. Wang, Z. Huang, and B. Karihaloo, "Size-dependent effective elastic constants of solids containing nano-inhomogeneities with interface stress," Journal of the Mechanics and Physics of Solids, Vol. 53, No. 7, pp. 1574-1596, 2005.

[15] Y. Pinyochotiwong, J. Rungamornrat, and T. Senjuntichai, "Rigid frictionless indentation on elastic half space with influence of surface stresses," International Journal of Engineering Science, Vol. 71, pp. 15-35, 2013.

[16] G. Jing, H. Duan, X. Sun, Z. Zhang, J. Xu, Y. Li, J. Wang, and D. Yu, "Surface effects on elastic properties of silver nanowires: contact atomic-force microscopy," Physical Review B, Vol. 73, No. 23, pp. 235409-1-235409-6, 2006.

[17] T.B. Nguyen, J. Rungamornrat, T. Senjuntichai, and A.C. Wijeyewickrema, "FEMSGBEM coupling for modeling of mode-I planar cracks in three-dimensional elastic media with residual surface tension effects," Engineering Analysis with Boundary Elements, Vol. 55, No. 6, pp. 40-51, 2015.

[18] J. Rungamornrat, P. Tuttipongsawat, and T. Senjuntichai, "Elastic layer under axisymmetric surface loads and influence of surface stresses," Applied Mathematical Modelling, Vol. 40, pp. 1532-1553, 2016.

[19] C. Wu, "The effect of surface stress on the configurational equilibrium of voids and cracks," Journal of the Mechanics and Physics of Solids, Vol. 47, No. 12, pp. 24692492, 1999.

[20] C. Wu, and M. Wang, "The effect of crack-tip point loads on fracture," Journal of the Mechanics and Physics of Solids, Vol. 48, No. 11, pp. 2283-2296, 2000.

[21] C. Wu, and M. Wang, "Configurational equilibrium of circular-arc cracks with surface stress," International Journal of Solids and Structures, Vol. 38, No. 24-25, pp. 42794292, 2001.

[22] G. Wang, X. Feng, T. Wang, and W. Gao, "Surface effects on the near-tip stresses for mode-I and mode-III cracks," Journal of Applied Mechanics, Vol. 75, No. 1, pp. 011001-1-011001-5, 2008.

[23] Q.H. Fang, Y. Liu, Y.W. Liu, and B.Y. Huang, "Dislocation emission from an elliptically blunted crack tip with surface effects," Physica B: Condensed Matter, Vol. 404, No. 20, pp. 3421-3424, 2009.

[24] X.L. Fu, G.F. Wang, and X.Q. Feng, "Surface effects on the near-tip stress fields of a mode-II crack," International Journal of Fracture, Vol. 151, No. 2, pp. 95-106, 2008.

[25] X.L. Fu, G.F. Wang, and X.Q. Feng, "Surface effects on mode-I crack tip fields: A numerical study," Engineering Fracture Mechanics, Vol. 77, No. 7, pp. 1048-1057, 2010.

[26] G. Wang, and Y. Li, "Influence of surface tension on mode-I crack tip field," Engineering Fracture Mechanics, Vol. 109, pp. 290-301, 2013.

[27] E. Oh, J.R. Walton, and J. Slattery, "A theory of fracture based upon an extension of continuum mechanics to the nanoscale," Journal of applied mechanics, Vol. 73 No. 5, pp. 792-798, 2006. 
[28] C. Kim, P. Schiavone, and C. Ru, "Analysis of a mode-III crack in the presence of surface elasticity and a prescribed non-uniform surface traction," Zeitschrift für angewandte Mathematik und Physik, Vol. 61, No. 3, pp. 555-564, 2010.

[29] C. Kim, P. Schiavone, and C. Ru, "Analysis of plane-strain crack problems (mode-I \& mode-II) in the presence of surface elasticity," Journal of Elasticity, Vol. 104 No. 1-2, pp. 397-420, 2011.

[30] C. Kim, P. Schiavone, and C. Ru, "The effect of surface elasticity on a Mode-III interface crack," Archives of Mechanics, Vol. 63, No. 3, pp. 267-286, 2011.

[31] T. Sendova, and J.R. Walton, "A new approach to the modeling and analysis of fracture through extension of continuum mechanics to the nanoscale," Mathematics and Mechanics of Solids, Vol. 15, No. 3, pp. 368-413, 2010.

[32] C. Kim, C. Ru, and P. Schiavone, "A clarification of the role of crack-tip conditions in linear elasticity with surface effects," Mathematics and Mechanics of Solids, Vol. 18 No. 1, pp. 59-66, 2013.

[33] H. Nan, and B. Wang, "Effect of residual surface stress on the fracture of nanoscale materials," Mechanics Research Communications, Vol. 44, pp. 30-34, 2012.

[34] H. Nan and B. Wang, "Effect of crack face residual surface stress on nanoscale fracture of piezoelectric materials," Engineering Fracture Mechanics, Vol. 110, pp. 68-80, 2013.

[35] J.R. Walton, "Plane-strain fracture with curvature-dependent surface tension: mixedmode loading," Journal of Elasticity, Vol. 114, No. 1, pp. 127-142, 2014.

[36] J.R. Barber, Elasticity: Solid Mechanics and Its Applications, Kluwer Academic Publishers, Dordrecht, Netherlands, 1992.

[37] J.P. Hirth, and J. Lothe, Theory of Dislocations, John Wiley \& Son, New York, United States, 1982.

[38] H.C. Tran, A Computational Procedure for Analysis of Fractures in Two-Dimensional Multi-Field Media, Thesis (PhD), University of Texas, Austin, United States, 2010.

[39] R.E. Miller, and V.B. Shenoy, "Size-dependent elastic properties of nanosized structural elements," Nanotechnology, Vol. 11, No. 3, pp. 139-147, 2000. 